\title{
The Application of Decorative Jinsha Gold Decoration on the New
}

\section{Chinese Style Furniture}

\author{
Jing Zeng, Chunling Gu
}

College of Forestry, Sichuan Agricultural University, Wenjiang, 611130,China

Keywords: Jinsha gold; decorative pattern; new Chinese style furniture design

\begin{abstract}
Jinsha Ruins unearthed gold unique style, distinctive features, culture, art, design of high value. The unique characteristics and connotation of the decorative patterns can be the object of the new Chinese style furniture design. This paper uses the method of case analysis and investigation methods of the Jinsha site in the gold decoration application of new Chinese style furniture design analysis. Image characteristics of decorative patterns of Jinsha Site gold extraction with first from Jinsha gold in the typical "Sunbird" gold and gold, gold parses Jinsha Ruins decorative patterns with the sun worship and connotation; with modern design nationalization and personalized aesthetic taste of modern science, returning to nature and the pursuit of simplicity for refining based on the proposed four methods, Jinsha gold refined decoration patterns: direct references, exaggerated changes, abstraction, decomposition and combination, extracted from Jinsha gold typical patterns, obtain new design elements; and then summed up the new Chinese style furniture decorative pattern parts, pointed out the direction for the use of Jinsha gold decorative patterns in the new Chinese style in furniture. Using the research method of this study can provide new design elements for the new Chinese style furniture design, promote the inheritance and innovation of Jinsha gold decorative patterns in the new Chinese furniture, inherit and develop the ancient Shu culture.
\end{abstract}

\section{Background}

$\mathrm{Hu}$ Jingchu pointed out in the article "the development trend of the furniture industry in the junior middle school in the 21st century", since entering the new century, under the impetus of the industrial revolution, the world has entered the era of design. Design can not only revitalize the economy, expand exports, but also can increase the added value of products, improve competitiveness, so the furniture industry is also the case."

Jinsha site is one of the most important sites in the pre Qin period in China. It has been proved that the Chengdu plain is the center of the civilization of the upper reaches of the Yangtze River, and is an important part of the Chinese civilization. The discovery of the Jinsha site, greatly expand the connotation and extension of the ancient Shu culture, is of great significance to the study of Shu culture origin, development and decline of the reproduction of the ancient Shu Kingdom of glory, the resurrection of a history of loss, reveals a deep sleep for more than 3000 years of Ming Dynasty of ancient literature.

Nowadays, the culture tends to be cosmopolitan and diverse, and people are more concerned with the design of national and individual. Furniture design while absorbing the essence of traditional culture, but also shoulder the responsibility of heritage culture. In the new Chinese style furniture design, should pay attention to the original spirit and cultural heritage, this paper hopes to Jinsha gold decoration patterns in the application of new Chinese style furniture, development and 
inheritance of Bashu culture.

Modern living conditions have greatly improved and social environmental protection consciousness enhancement, the people more preference and longing for nature, the Nordic Scandinavia design schools which rise, emphasizing the application of natural materials and natural color, produced extensive and profound influence in the world. Based on this, the designers in order to meet the modern people advocating natural psychological factors, and continue to work in the "return to nature", using a variety of design techniques to enable people to think about nature, feel the natural. Therefore, return to nature, nature, and Jinsha gold decorative patterns of the one direction of re creation.

\section{Jinsha Introduced Gold}

Jinsha site is one of the most important sites in the pre Qin period in China. It has been proved that the Chengdu plain is the center of the civilization of the upper reaches of the Yangtze River, and is an important part of the Chinese civilization. The discovery of the Jinsha site, greatly expand the connotation and extension of the ancient Shu culture, is of great significance to the study of Shu culture origin, development and decline of the reproduction of the ancient Shu Kingdom of glory, the resurrection of a history of loss, reveals a deep sleep for more than 3000 years of Ming Dynasty of ancient literature. As one of the gold Jinsha Jinsha site culture in the most representative of the cultural relics, the pattern has profound cultural connotation and artistic value of high aesthetic characteristics, unique, occupies an important position in Chinese civilization history.

Jinsha gold with culture, art and design high value. Flying flow Jinsha gold decorative patterns, the drift, spirited original romantic temperament full of life vitality and artistic passion, has expanded the meaning of new Chinese style furniture design. However, on Jinsha gold books and articles, mostly from the perspective of archaeology, history, art characteristic to study, there is little research from the perspective of furniture design.

More than and 30 pieces of gold unearthed cultural relics unearthed from the site of Jinsha is the site of the most unique style and distinctive features of their own. These include gold, gold, gold mask with round gold, gold, gold frog shaped horn etc.. In addition to the golden mask and Sanxingdui bronze mask in the same style on the outside, other types of gold are unique to the Jinsha, is gold, gold foil hammering, is very rich in species.

In all Chinese unearthed ancient cultural relics in the worship of the sun pattern, no doubt to the "Sunbird" gold pattern is the most exquisite, it expresses the connotation with a pregnant meaning. The sun bird gold diameter $12.5 \mathrm{~cm}, 5.29 \mathrm{~cm}$ in diameter, thickness of $0.02 \mathrm{~cm}$, weight 20 grams. Outline a circular pattern divided into two layers, using the form through the air. The inner pattern of the twelve curved teeth of the distribution of isometric, decorated with a clockwise rotation. The outer pattern consists of four equally spaced birds. The birds were flying, long legs, flying direction and the inner pattern of the opposite. Twelve clockwise light and four reverse flying bird, the expression of the ancient people's pursuit of life, longing for light. Full of vigor and vitality of the "Sun Bird" to give people inspiration and great power, embodies the diligence and wisdom of ancient Shu kingdom. The whole piece of cultural relics shows the outstanding craftsmanship of the ancient Shu people, which is the representative of the brilliant achievements of the ancient Shu Jin craft.

August 2005, "Sun Bird gold appeared attracted by the State Administration of cultural heritage, was elected as the first sign of cultural relics protection Chinese. According to the State Administration of cultural heritage interpretation, "Sun Bird" is the heritage of material and spirit: it is just a matter of gold foil, convey the spirit is as bright as gold. The State Bureau of cultural relics 
experts identified, "Sun Bird" pattern reflects the "ancient people" harmony "philosophy". "People are the center of the world", advocated the harmonious coexistence of man and nature, the transfer is the humanist spirit; national cultural relics bureau also pointed out that the "four birds fly around the sun, reflects the ancestors of the pursuit of freedom, beauty, moral unity upward". This "freedom, happiness, harmony, upward", that is, the spirit of the ancient Shu culture yearning. [2]

Unearthed in Chengdu Jinsha Ruins of gold, and exquisitely carved patterns in gold belt, is a very amazing artifacts, is of great significance in the history of archaeology.

The circle shaped gold crown with a circle diameter of 19.6 - $19.9 \mathrm{~cm}$, bandwidth $2.68-2.8 \mathrm{~cm}$, thickness of $0.02 \mathrm{~cm}$, weight of 44 grams. At the same time with the other gold unearthed in the production process of the same department, hammering forming, and the carving technique, on the surface with description of subtle gold rich meaning of decoration patterns.[3]

This is the most amazing golden belt pattern, with exquisite carving craft, depict on the surface with golden delicious, even subtle performance is very clear, give a person with smooth lines and vivid feeling. On the whole by the four groups of the same pattern, the use of symmetrical layout, concise and vivid expression, the image is singular, rich in meaning, showing a wonderful creative ideas and superb characterization techniques. Each pattern respectively to describe a fish, a bird, an arrow and a circle. The most prominent is the long rod arrow across the pattern, which is now thick long, after the tail shaft, shoot birds and fish, birds across the neck into the head first. The bird form thick neck hook beak, a long tail, legs and claws outstretched wings to jumping, eyes bright, looks very lively.

The golden belt on each pattern, but also depicts the design of the wonderful double circle pattern. The circle diameter of about $2 \mathrm{~cm}$, the outer contour of two rotary lines, and two symmetrical double spiral pattern composed of small circle, small circle in each on and have a short thick stripes, the abstract approach to the ingenious combination, thus forming a circle like day and like people or animal face patterns. There are a total of four sets of patterns on the gold belt, so that the double circle pattern also depicts the four. From the perspective of style, the symbol of the round day with the human face or animal face double circle pattern also gives a sense of unrestrained, in the design of the combination has a special meaning. From the overall design layout, one double circle pattern that is located in the golden belt in front of the central, the double circle pattern as the center, in which both sides are symmetrically arranged across the neck of the arrow into the bird head pattern, bird head and head toward the double circle pattern. This seems to tell us that the symbol round day and human face or the beast double circle pattern in the whole picture in the dominant position, with golden pattern rich meaning of double circle pattern, should reflect the ancient Shu people worship the sun concept, golden belt of the decoration patterns, are extremely rich in meaning. Both describe style, technique, or moral conception, it is greatly have great originality.

\section{Gold Pattern Extraction Method}

Four methods of Jinsha gold refined modern decorative patterns: direct borrowing and exaggerated changes, abstraction, decomposition and combination of gold, Jinsha typical patterns extracted, parsing out the new design elements; and concludes the new Chinese style furniture patterns and decorative parts, decorative parts of Jinsha gold decorative patterns for new Chinese style furniture.

Direct adduction is the Jinsha site in some gold beautiful patterns, cultural implication of the decorative patterns directly extracted, to maintain the original flavor of antique patterns[4], direct reference is the most basic pattern extraction method. Jinsha Ruins gold decorative patterns and 
focus on the dynamic momentum image, everywhere to show the changes in the strength, and the linear speed, using a simplified simple image to express life style of this period in different poses and with different expressions, simple patterns, the morphological characteristics of modern patterns is brief and concise easy to accept by modern people, but also to facilitate processing. "The true tradition is the product of constant progress, the essence of which is movement, not rest," he said. Tradition should push people forward." By refining the direct reference method Jinsha gold decorative patterns, patterns of artistic design and prototype to retain cultural charm with modern design concept of nationalization.

Change is exaggerated exaggerated refined prominent features on Jinsha gold decorative patterns properly master view of exaggeration and change [5], to strengthen the original image features and increase the decorative effect. Han people generally like to enlarge the image of the characteristics of the part, omitting a large number of non characteristic details, so that the characteristics of the part to be prominent, so the exaggeration and deformation is commonly used modeling techniques chu. We can combine the traditional patterns of exaggeration change methods and modern exaggerated refined expression, such as exaggeration, hyperbole and exaggeration, and local dynamic change of genre painting expression [6], such as constructivism, expressionism, so that the Jinsha gold decorative patterns can create new patterns. At the same time, we can make some simple patterns Jinsha gold decorative patterns in one direction as axis, repeated arrangement, upside down together, such as tensile and flexural deformation of the. This extraction method is based on understanding the changes of exaggerated human factors on the subjective of Jinsha gold decorative patterns of exaggeration and change, it conforms to the modern design concept of personalized.

abstract generalization is based on the understanding of the Jinsha Ruins gold decoration patterns of spirit, through the method of abstract changes, on the Jinsha site for the two other gold decorative patterns combined with the deformation curve or straight line, the pattern is terse, yet the original patterns contained that is abstract, concise modern beauty. With the environmental protection, ecology, sustainable development, and so on, people began to advocate.

\section{Furniture Decoration}

With the development of society, consumers pay attention to the function and shape of the furniture at the same time, also hope that the furniture contains history and culture can make people empathize with Lenovo, reasonable decorate parts so furniture designers need to choose the appropriate. How to carry on the decoration of furniture parts, Mr. Hu Jingchu once pointed out that "the decoration of furniture generally has two kinds: one kind is the combination of furniture and decorative elements to maintain the overall shape, combination of the original structure, is decorated with appropriate in important parts of the furniture. The other is to beautify the processing of the entire furniture structural parts in a prominent position."

1) The decorative parts of the new Chinese chairs are: the chair, armrest, soft bag, legs, sheathing and tooth plate etc. The chair is in the decorative parts of the highest frequency, followed by soft and handrails.

2) The main decorative parts of new Chinese style furniture's door and drawer panel, handle, leg and side and rail etc...The door panel is the highest frequency in the decorative parts, followed by the drawer panel and handle.

3) The decorative parts of new Chinese style furniture table furniture including desktop panel and rail, desk legs, a drawer panel, handle etc... Where is the decorative parts of rail in the highest frequency, followed by the top panel and the leg of the table. 
4) The decorative parts of the new Chinese bed furniture are: bed screen, bed, bed, bed leg circumference, sheathing etc... Among them, the frequency of the bed screen and the tail of the bed is the highest.

\section{Conclusion}

Reasonable decoration can not only beautify the furniture, but also to make furniture with more social value and cultural connotations, the new Chinese style furniture is such a style. Through the understanding of the content of the decorative patterns on the new Chinese style furniture, the understanding of the inner meaning of decorative patterns, and the recall of its historical elements, the resonance of the soul. Jinsha gold decorative patterns used in the new Chinese style furniture, should pay attention to its rationality, which ultimately achieve beautification effect of furniture. At the same time, it can highlight the cultural connotation of the new Chinese style furniture.

China's modern furniture design, China needs to contain the Chinese culture and the characteristics of the times of the new Chinese style furniture. At the same time, the modern people in the enjoyment of the sense of the times of practical, aesthetic, comfortable, but also the pursuit of the humanities to highlight the furniture, to meet their needs for national cultural connotations. So when inheriting and absorbing the Jinsha Ruins gold decorative patterns, not only strengthen the shape of the reference, we should pay more attention to understand the profound meaning behind the patterns, namely take the meaning of the spirit. So as to reflect the national culture and the national style in the design, so that in the design process can not be separated from the national characteristics, but also has the freedom to create space.

\section{Funds Project:}

Key Laboratory of Wood Industry and Furniture Engineering of Sichuan Provincial Colleges and Universities

\section{References}

[1] Mo Gang Gang. Art morphology [M]. Ling Jiyao, translated by Jin Yana. Sanlian bookstore, 1986.

[2] Yu Xiao Hong. Study on the modern application of carved decoration patterns of Ming and Qing furniture [D]. Beijing: Beijing Forestry University.2006109-110.

[3] Heinrich. Chinese art style. Translated by [M]. Pan Yaochang. Renmin University of China press, 2004.

[4] Zhuang Ziping. Creative application of classic decorative patterns [M]. Jilin Fine Arts Publishing House.2010,8-9.

[5] Zhuang Ziping. Creative application of classic decorative patterns [M]. Jilin Fine Arts Publishing House.2010,8-9.

[6] Hu Jingchu, Dai. An introduction to furniture design [M]. Beijing: China Forestry Publishing House, 1999. 